\title{
An unusual case of schwannomatosis with bilateral maxillary sinus schwannomas and a novel SMARCB1 gene mutation
}

\author{
Jamie Toms, MD, ${ }^{1}$ Jason Harrison, MD, PhD, ${ }^{1}$ Hope Richard, MD, PhD, ${ }^{2}$ Adrienne Childers, MD, ${ }^{3}$ \\ Evan R. Reiter, MD, ${ }^{3}$ and Robert S. Graham, MD'
}

Departments of ${ }^{1}$ Neurosurgery, ${ }^{2}$ Pathology, and ${ }^{3}$ Otolaryngology, Virginia Commonwealth University, Medical College of Virginia, Richmond, Virginia

\begin{abstract}
Schwannomas are benign tumors that arise from Schwann cells in the peripheral nervous system. Patients with multiple schwannomas without signs and symptoms of neurofibromatosis Type 1 or 2 have the rare disease schwannomatosis. Tumors in these patients occur along peripheral nerves throughout the body. Mutations of the SMARCB1 gene have been described as one of the predisposing genetic factors in the development of this disease. This report describes a patient who was observed for 6 years after having undergone removal of 7 schwannomas, including bilateral maxillary sinus schwannomas, a tumor that has not been previously reported. Genetic analysis revealed a novel mutation of c.93G $>\mathrm{A}$ in exon 1 of the SMARCB1 gene.
\end{abstract}

http://thejns.org/doi/abs/10.3171/2015.4.SPINE15192

KEY WORDS schwannomatosis; SMARCB1; peripheral nerve sheath tumor; perinasal schwannoma; oncology

$\mathrm{S}$ CHWANNOMAS are tumors arising from Schwann cells, and they ordinarily occur as a solitary encapsulated mass ${ }^{23}$ Schwannomatosis is a rare condition, characterized by the development of numerous tumors without the involvement of the vestibular nerve. The most common presenting symptom in these patients is pain..$^{9-11,14}$

Schwannomatosis is the third major form of neurofibromatosis. ${ }^{11,18,22}$ The incidence of this disease is 0.58 cases per 1 million people, ${ }^{4,14}$ with most cases occurring during the 3rd-6th decade of life. There does not seem to be sex or racial predisposition to the development of schwannomatosis. ${ }^{10,12}$ Most individuals have new mutations. Inherited cases account for $10 \%-15 \%$ of cases. ${ }^{22}$ A mutation of the SMARCBI gene, a potent tumor suppressor gene also involved in rhabdoid tumor development, has been shown to play a role in the development of schwannomatosis, in both sporadic and familial cases. ${ }^{6,10,19}$

\section{Case Report}

A 20-year-old African American woman was transferred to our facility with a 1-year history of lower-extremity pain, weakness, and sensory loss. She stated that her weakness progressed to the point that she could no longer walk, and she had lost bowel and bladder control 1 month earlier. The patient had no family history of central nervous system tumors, neurofibromatosis Type 1 or 2 , or schwannomatosis.

On physical examination, the patient was found to have diffuse weakness and sensory loss bilaterally in her lower extremities. Her lower-extremity reflexes were very brisk, and she had clonus bilaterally. On MRI, an intradural extramedullary mass posterior to the spinal cord extended from the midportion of T-6 to T-8 (Fig. 1). There was also a homogeneous, uniformly enhancing intradural extramedullary mass found at the level of L3-4 (Fig. 2). Imaging of the rest of the neuraxis was significant only for a small asymptomatic 5-mm schwannoma in the right Meckel's cave.

A T6-8 laminectomy revealed a tense dura, which was incised. The dura was then opened from T-6 to T-8. A large $3.3 \times 1.8 \times 1.0-\mathrm{cm}$ mass was found on the left side of the spinal canal, displacing the cord anteriorly and to the right. This mass extended from T-7 to T-8 and was firm in consistency and pale in color with a plane of arachnoid 

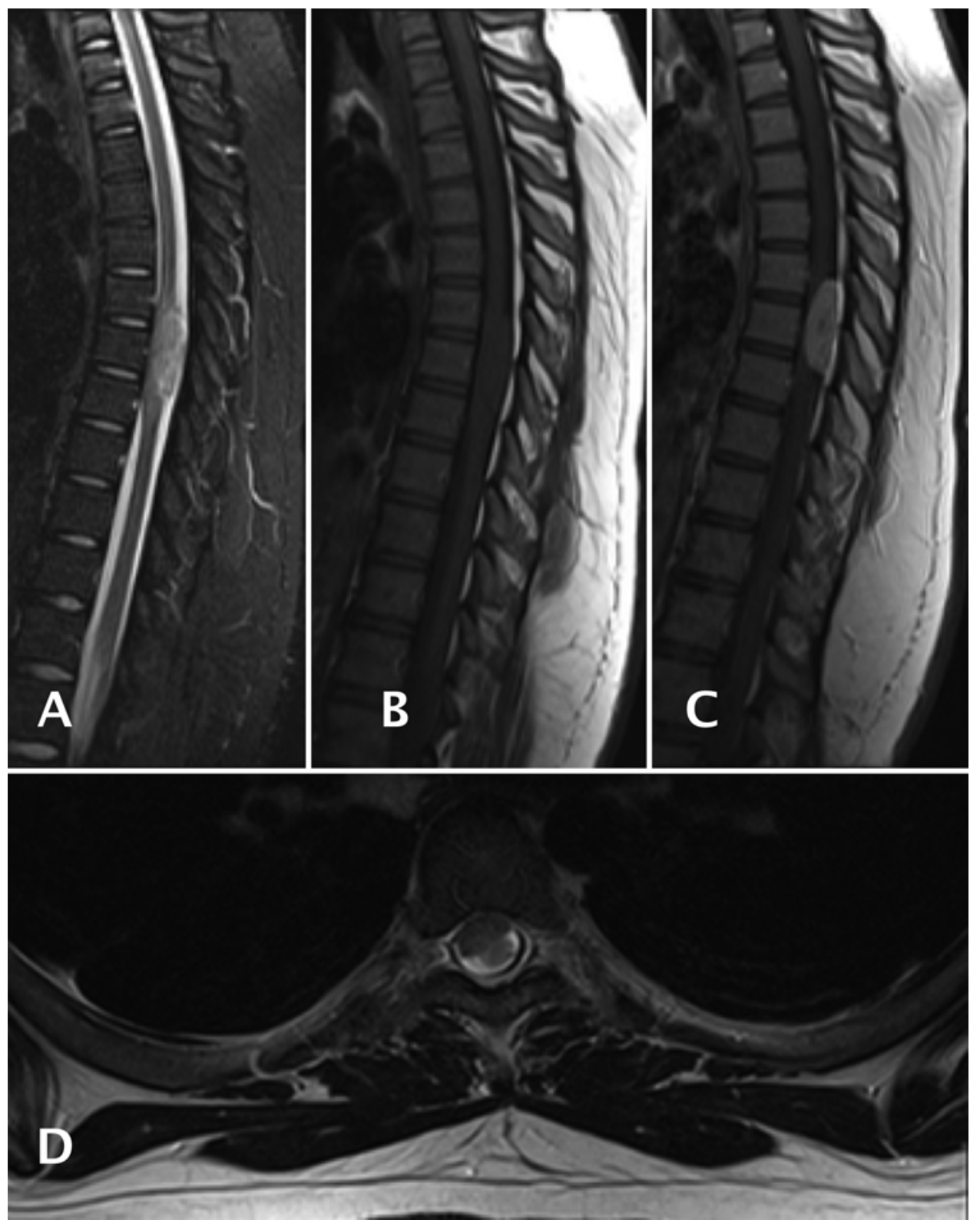

FIG. 1. Magnetic resonance images. A: Sagittal T2-weighted image of the thoracic spine showing a hyperintense mass. B: Sagittal T1-weighted image of the thoracic spine showing an isointense mass. C: Sagittal T1-weighted image of the thoracic spine with contrast showing a contrast-enhancing mass. D: Axial T2-weighted image of the thoracic spine showing an intradural extramedullary lesion. There is also cord edema and some slight dilation of the central canal.

separating the mass from the spinal cord. Dorsal roots appeared to be incorporated in the mass at numerous levels. There were no complications during the procedure, and pathology reports indicated that the mass was a cellular schwannoma (Fig. 3A).

Postoperatively, the patient was transferred for inpatient spinal cord injury rehabilitation. At the 2-month followup, she had regained lower-extremity function and control of her bowel and bladder with marked improvement in her sensation. The physician and patient decided at that point that she should undergo excision of the second lesion to prevent the mass from causing problems similar to those caused by her thoracic lesion. After much counseling and discussion with the patient, a separate procedure was then carried out to excise the L3-4 lesion. Histological evaluation of this mass revealed a schwannoma with increased mitotic activity and focal necrosis (Fig. 3B).

The patient had a full recovery from her 2 spine surgeries. However, 6 years after her initial presentation, she returned to the clinic with paresthesias and numbness of her left lower extremity. MRI at that time showed 3 separate lesions that had not been noted on previous images: a large, atypically heterogeneous enhancing lesion in the right psoas muscle adjacent to the right side of L4-5 (Fig. $4 \mathrm{~A}-\mathrm{C})$, a lesion at the inferior aspect of the sacrococcygeal junction pushing slightly on the posterior wall of the 

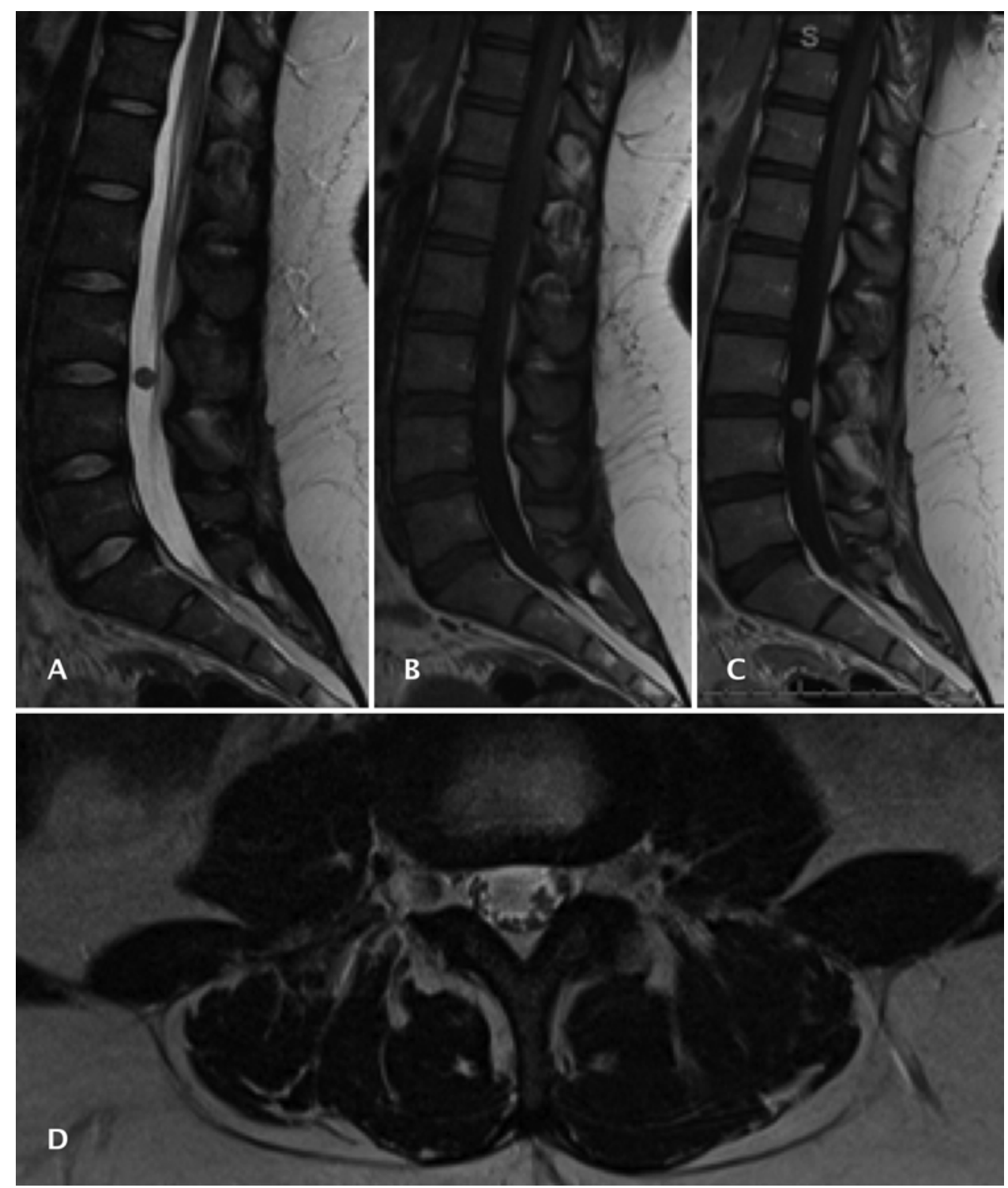

FIG. 2. Magnetic resonance images. A: Sagittal T2-weighted MR image of the lumbar spine showing a hypointense mass. B: Sagittal T1-weighted image of the lumbar spine showing a mildly hyperintense mass. C: Sagittal T1-weighted MR image of the lumbar spine with contrast showing a contrast-enhancing mass. D: Axial T2-weighted MR image of the lumbar spine showing an intradural extramedullary lesion.

rectum, and a lesion located in the left sciatic notch (Fig. 5). These lesions were not present on the earlier lumbar MR images.

The sciatic notch lesion was identified as the symptomatic lesion. However, given their accessibility through a single gluteal incision, both the sciatic notch and sacrococcygeal were excised. Both lesions were well demarcated and easily removed from the nerve sheath capsules. Again, histological analysis showed both tumors to be schwannomas (Fig. 3C). Genetic testing at that time indicated that the patient possessed a somatic mutation at c. $93 \mathrm{G}>\mathrm{A}$ in exon 1 of the SMARCBI gene.

Again, the patient recovered well from her procedure, and her symptoms abated. At follow-up, the patient had noted hyponasal speech and complained of increased drainage from her nose. An MR image showed a stable 5-mm schwannoma in the right Meckel's cave, dural thickening of the right falx in the frontal area, and a multilobulated, contrast-enhancing, cystic mass expanding the left maxillary and right maxillary and sphenoid sinuses. The mass did not invade the adjacent bone (Fig. 6). The patient had been having increasing nasal congestion and clear drainage bilaterally. A bilateral nasal endoscopy was performed [using a flexible endoscope] and showed narrow nasal cavities bilaterally with a pale gray mass in the right posterior nasal cavity.

The patient was returned to the operating room for endoscopic removal of her maxillary sinus masses. An endoscope was introduced into the patient's left nasal passage, and the lateral nasal wall was noted to be protruding medially. The uncinate process was then debrided, revealing a cystic capsule within the maxillary sinus. The cap- 

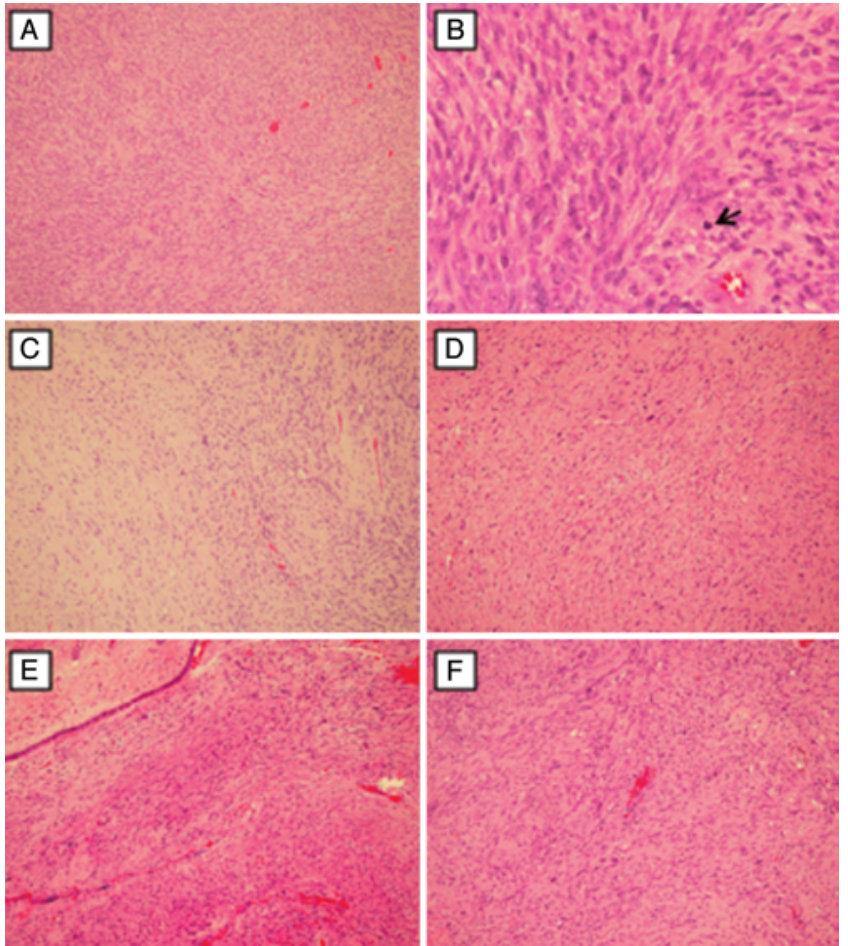

FIG. 3. Histopathological analysis. A: T6-8 cellular schwannoma showing increased cellularity and focal Verocay body formation. B: L-3 schwannoma with atypical features including increased cellularity and mitotic activity (arrow). C: Left sacral schwannoma with typical biphasic histology. D and E: Left (D) and right (E) maxillary sinus schwannomas. F: Right psoas schwannoma. Original magnification $\times 10(A, C$, $\mathrm{D}-\mathrm{F}) ; \times 40(\mathrm{~B})$. Figure is available in color online only.

sule was then decompressed. Once the capsule had been decompressed, the remainder of the cystic structure was carefully removed (Fig. 7). Attention was then turned to the right nasal passage where a similar procedure was performed. Histological analysis indicated the lesions to be schwannomas (Fig. 3D and E).

Four weeks later, the patient returned to the operating room for removal of a large L-4 nerve sheath tumor in her right psoas muscle, which was also found to be a schwannoma (Fig. 3F). The patient tolerated the surgery very well and was discharged home from the hospital shortly after the procedure. Six weeks after surgery, the patient had recovered from surgery well. Because of her aggressive disease, further plans were made to follow up with the patient every 6 months with serial MRI. At her first 6-month appointment after her surgery, imaging showed a gross-total resection and no recurrence (Fig. 4D). She was also noted to have no new lesions at that time.

\section{Discussion}

Schwannomatosis is a rare disease characterized by the development of multiple schwannomas ${ }^{11,18,22}$ and meningiomas. ${ }^{14}$ The present case is a particularly aggressive case and is associated with a novel mutation in the SMARCBI gene. In addition, this case highlights the variety of locations that these lesions can be found including, and also previously not described, in the maxillary and sphenoid
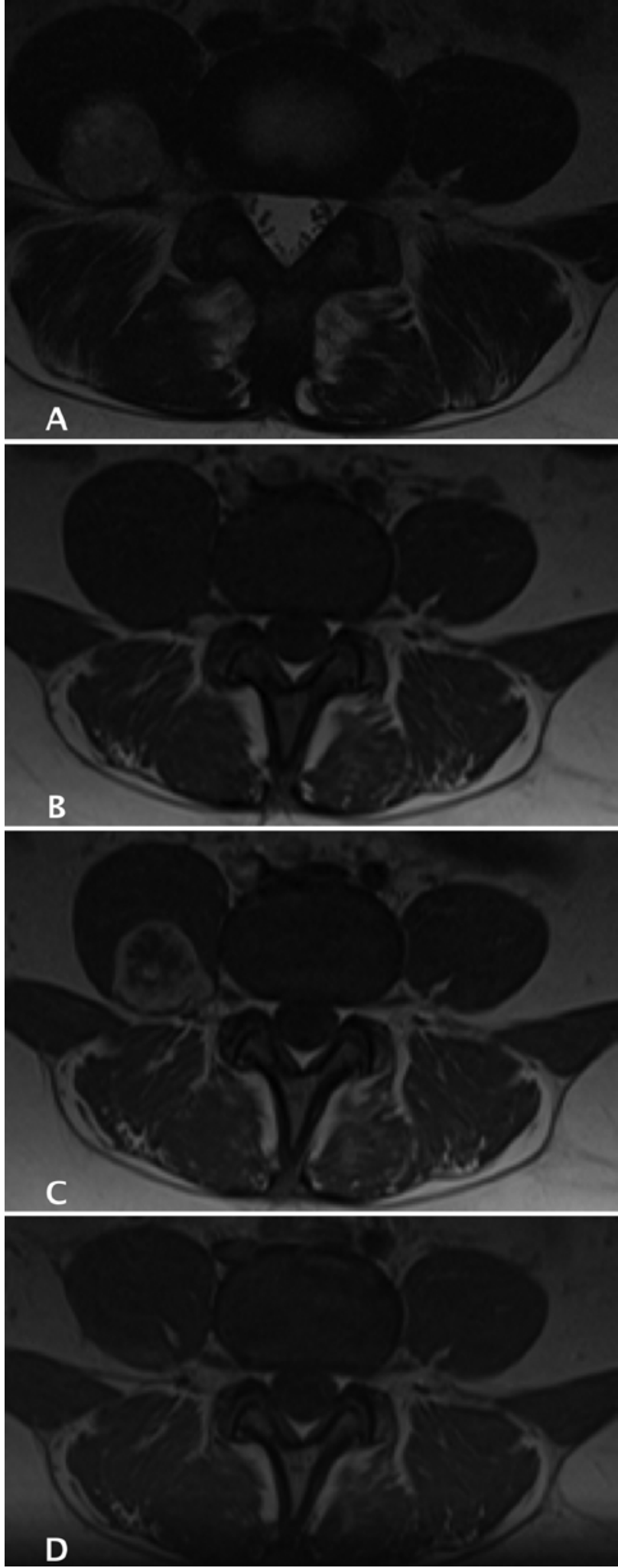

FIG. 4. Magnetic resonance images. A: Axial T2-weighted image showing a $2.4 \times 2.7-\mathrm{cm}$ heterogeneous hyperintense mass in the right psoas muscle. B: Axial T1-weighted image without contrast showing an isointense mass in the right psoas muscle. C: Axial T1-weighted image with contrast showing a contrast-enhancing mass in the right psoas muscle. D: Axial T1-weighted MR image with contrast obtained 6 months postoperatively, showing good resection and no recurrence. 

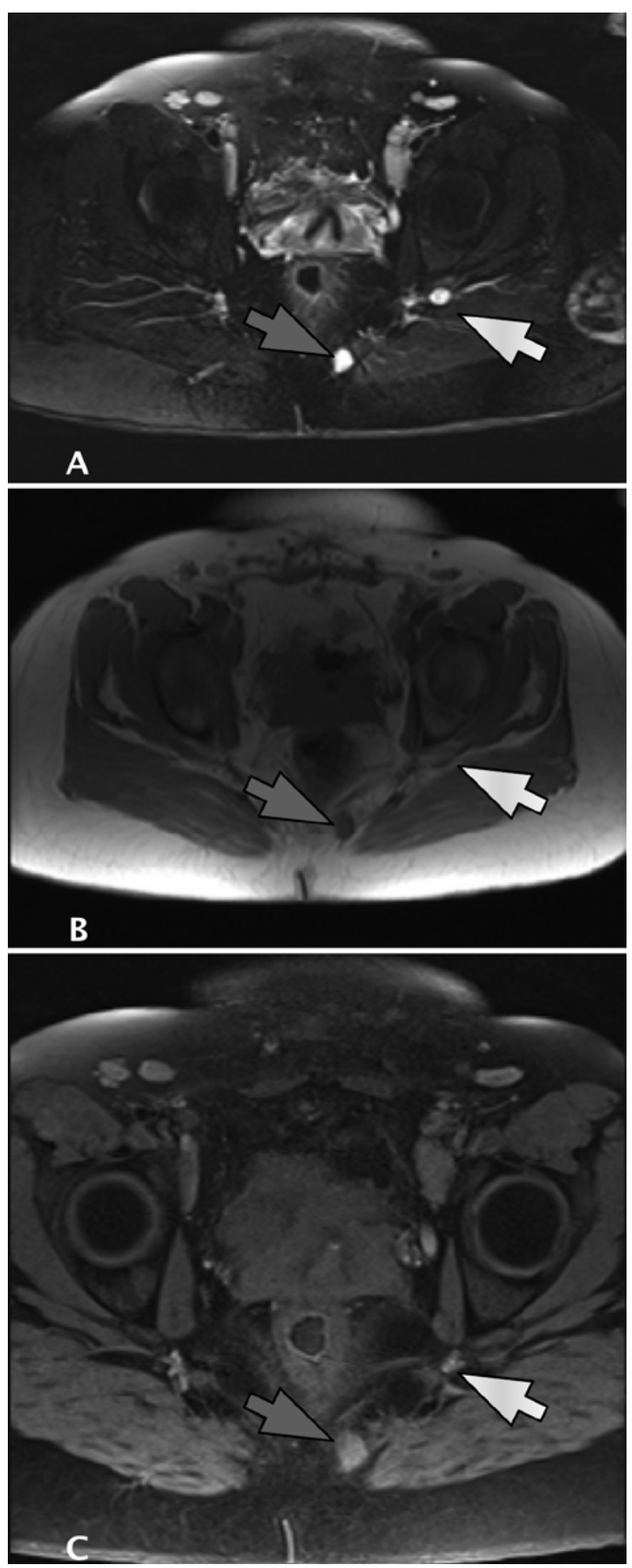

FIG. 5. Magnetic resonance images. A: Axial T2-weighted image with fat saturation showing a hyperintense $2.4 \times 1.4 \times 1.4-\mathrm{cm}$ mass at the left inferior aspect of the sacrococcygeal junction (gray arrow) and a 1.2$\mathrm{cm}$ hyperintense mass at the left sciatic notch (white arrow). B: Axial T1-weighted image without contrast showing an isointense mass at the sacrococcygeal junction (gray arrow) and left sciatic notch (white arrow). C: Axial T1-weighted image with contrast showing a contrastenhancing mass at the sacrococcygeal junction (gray arrow) and left sciatic notch (white arrow). sinuses. Coordination of multiple disciplines including neurosurgery, surgical oncology, otolaryngology, neurooncology, and genetics was necessary to optimize outcome for this case and to provide continued tumor surveillance.

Merker et al. found that $68 \%$ of patients with schwannomatosis experienced chronic pain. ${ }^{14}$ These patients can also have symptoms of paresthesias, weakness, and muscle atrophy. ${ }^{5}$ Our patient's presentation was no exception; she initially presented with bilateral weakness in her lower extremities. However, in contrast to the usual presentation, she also exhibited loss of bowel and bladder control, which was a result of late presentation and a severe spot of cord compression of the thoracic lesion.

Although peripheral nerve sheath tumors have a tendency to form in the head and neck, perinasal schwannomas are exceedingly rare,,$^{2,13,20}$ with only $4 \%$ of these tumors occurring in these locations. ${ }^{20}$ These tumors can arise from parasympathetic fibers of the sphenopalatine ganglion, sympathetic fibers of the carotid plexus, or branches of the ophthalmic and maxillary divisions of the trigeminal nerve. ${ }^{3}$ To our knowledge, this is the first reported account of a perinasal schwannoma occurring in a patient with schwannomatosis.

Another unique quality of this case is the patient's unique mutation of $S M A R C B 1$. The patient has a family history that is negative for neurofibromatosis Type 1 or 2 or schwannomatosis. The sporadic origin in this case is consistent with the approximately $90 \%$ of de novo mutations seen in schwannomatosis. ${ }^{8,21}$ This specific mutation, c. $93 \mathrm{G}>\mathrm{A}$ in exon 1 has not previously been described (Fig. 8). Similar gene mutations have, however, been reported in cases of rhabdoid tumors,,$^{1,7}$ but, to our knowledge, this mutation has not been previously described in the schwannomatosis literature.

The SMARCBI gene is a tumor suppressor gene located on chromosome 22.3,6,10 This gene spans 47,554 base pairs and includes 9 exons that code a protein that is a subunit of an adenosine triphosphate-dependent chromatin-remodeling complex that relaxes regions of chromatin and allows transcription to occur. Loss of this SMARCBI gene function results in chromosomal instability and polyploidy in rhabdoid tumors.,17 Mutations in this gene are also responsible for around $50 \%$ of familial schwannomatosis and $10 \%$ of sporadic schwannomatosis. ${ }^{16}$ The exact mechanisms by which these mutations cause schwannomatosis are not well understood. Sestini et al. described a 4-hit mechanism involving the genes SMARCB1 and NF2 in the development of schwannomatosis. ${ }^{19}$ There have also been reports of LZTR 1 being involved in a large number of schwannomatosis cases. ${ }^{15,16}$ Hutter et al., after sequencing the schwannomatosis exome, suggested that even more gene mutations on chromosome 22 must exist to account for the cases of schwannomatosis when SMARCBI and LZTR1 are excluded. ${ }^{8}$ Zhang et al. reported that perhaps mutations in COQ6 play a role in schwannomatosis development. ${ }^{24}$

\section{Conclusions}

To our knowledge, we present the first case of paranasal schwannomas occurring in schwannomatosis as well 

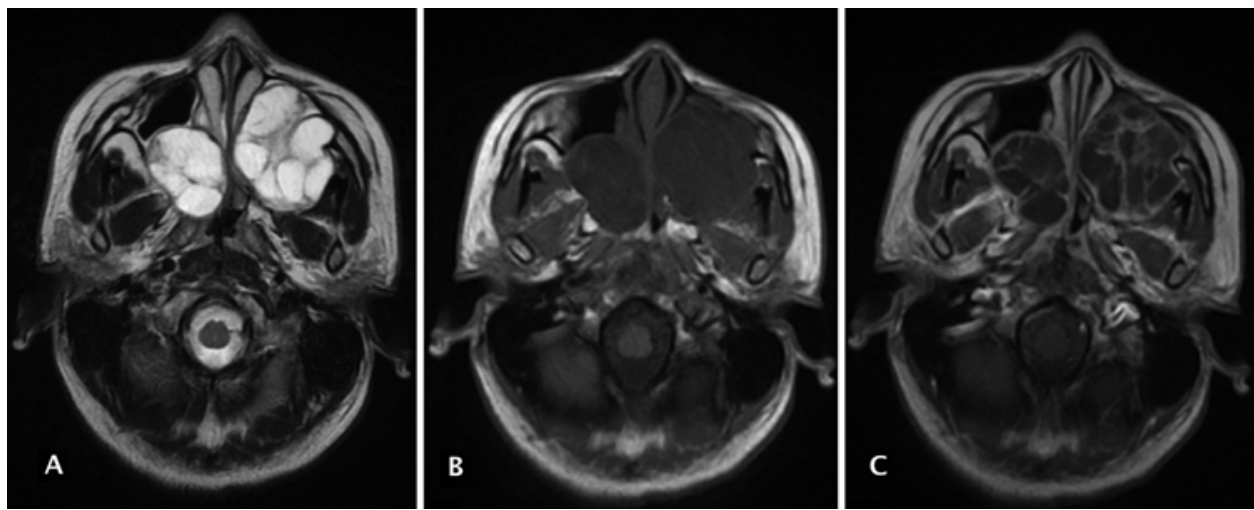

FIG. 6. Magnetic resonance images. A: Axial T2-weighted image of the head showing hyperintense cystic masses in bilateral maxillary sinuses. B: Axial T1-weighted image of the head without contrast showing masses in bilateral maxillary sinuses. C: Axial T1-weighted image of the head with contrast showing a multilobulated cystic mass in bilateral maxillary sinuses.
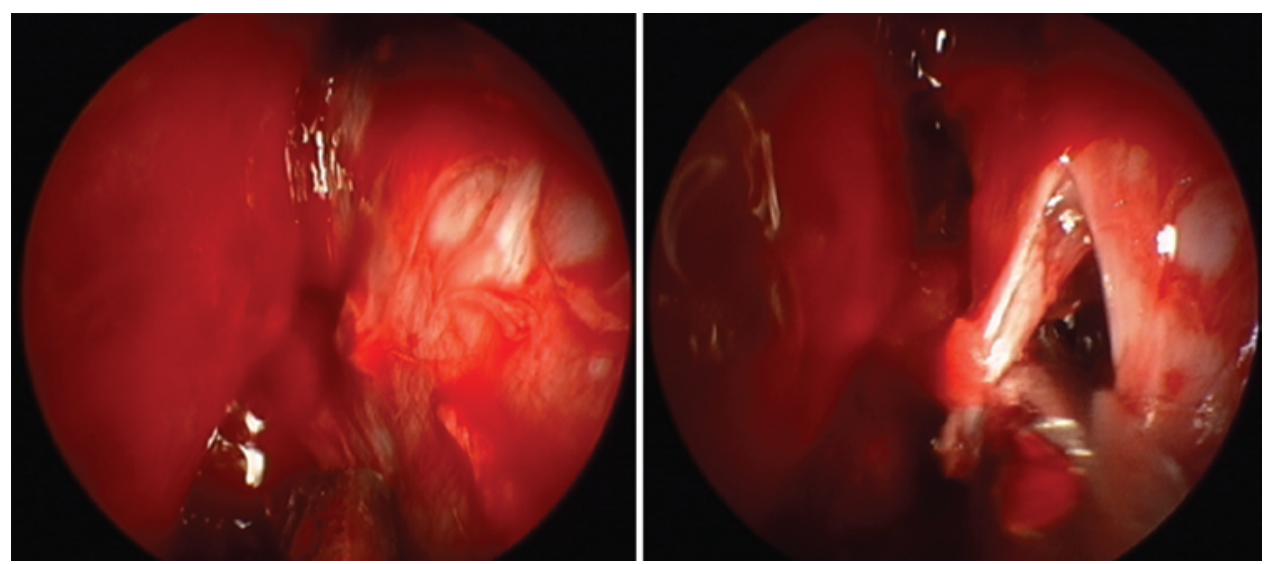

FIG. 7. Endoscopic view of a left-sided cyst protruding from the maxillary sinus (left) and cyst decompression (right). Figure is available in color online only.

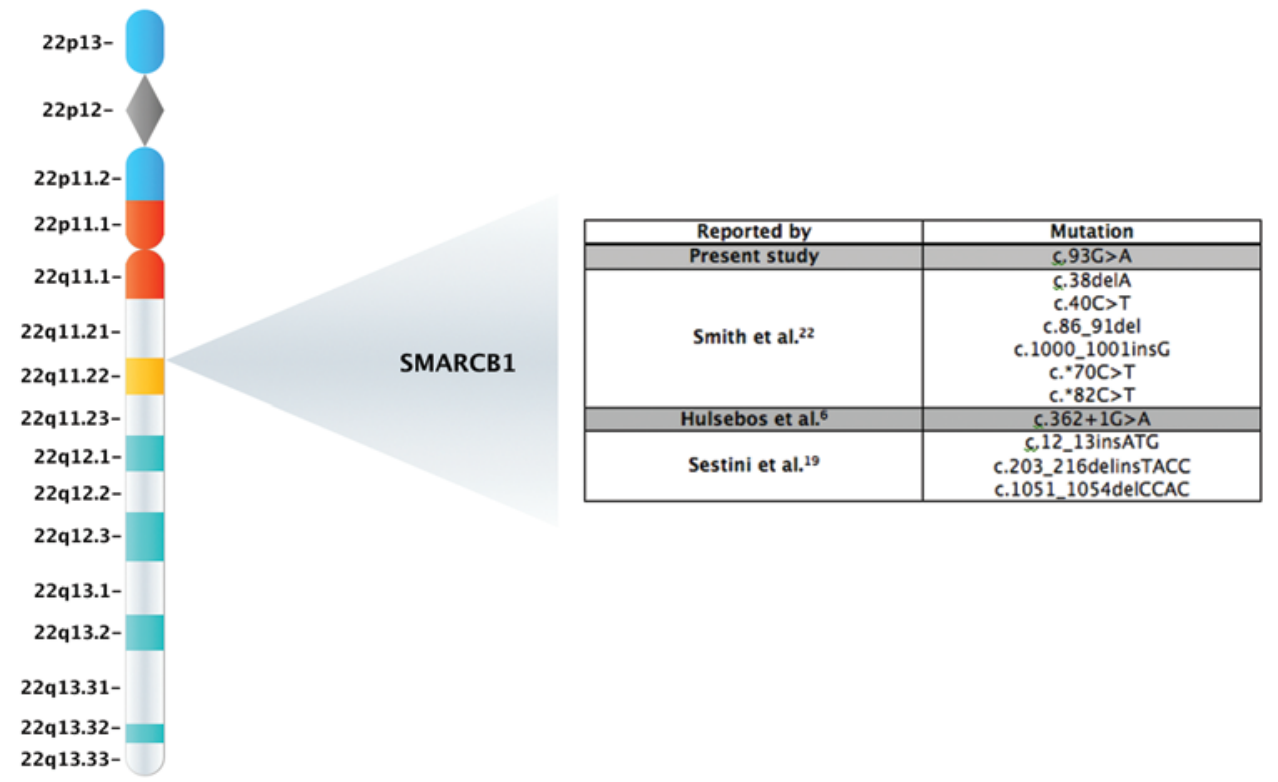

FIG. 8. Chromosome 22 with reported SMARCB1 mutations with approximate location of SMARCB1 on the chromosome. Multiple mutations have been reported in the past. A similar mutation to $c .93 \mathrm{G}>\mathrm{A}$ has only been reported previously in rhabdoid tumors. Figure is available in color online only. 
as the first reported mutation of c.93G $>\mathrm{A}$ in exon 1 of the SMARCB1 gene. This case demonstrates the complexity of schwannomatosis treatment, symptomatology, and genetics.

\section{References}

1. Carroll SL: Molecular mechanisms promoting the pathogenesis of Schwann cell neoplasms. Acta Neuropathol 123:321348,2012

2. Chacko AG, Natarajan MS, Prabhu K, Chacko G: Schwannoma of supraorbital nerve presenting as a subfrontal tumor. Neurol India 62:96-98, 2014

3. Forest F, David A, Arrufat S, Pierron G, Ranchere-Vince D, Stephan JL, et al: Conventional chondrosarcoma in a survivor of rhabdoid tumor: enlarging the spectrum of tumors associated with SMARCB1 germline mutations. Am J Surg Pathol 36:1892-1896, 2012

4. Gonzalvo A, Fowler A, Cook RJ, Little NS, Wheeler H, McDonald KL, et al: Schwannomatosis, sporadic schwannomatosis, and familial schwannomatosis: a surgical series with long-term follow-up. Clinical article. J Neurosurg 114:756762,2011

5. Huang JH, Simon SL, Nagpal S, Nelson PT, Zager EL: Management of patients with schwannomatosis: report of six cases and review of the literature. Surg Neurol 62:353-361, 2004

6. Hulsebos TJM, Kenter S, Siebers-Renelt U, Hans V, Wesseling P, Flucke U: SMARCB1 involvement in the development of leiomyoma in a patient with schwannomatosis. Am J Surg Pathol 38:421-425, 2014

7. Hulsebos TJM, Kenter S, Verhagen WIM, Baas F, Flucke U, Wesseling P: Premature termination of SMARCB1 translation may be followed by reinitiation in schwannomatosis-associated schwannomas, but results in absence of SMARCB1 expression in rhabdoid tumors. Acta Neuropathol 128:439 448, 2014

8. Hutter S, Piro RM, Reuss DE, Hovestadt V, Sahm F, Farschtschi S, et al: Whole exome sequencing reveals that the majority of schwannomatosis cases remain unexplained after excluding SMARCB1 and LZTR1 germline variants. Acta Neuropathol 128:449-452, 2014

9. Jia Y, Kraus JA, Reddy H, Groff M, Wong ET: Polyradiculopathies from schwannomatosis. Open Neuroimaging J 5:9-13, 2011

10. Koontz NA, Wiens AL, Agarwal A, Hingtgen CM, Emerson RE, Mosier KM: Schwannomatosis: the overlooked neurofibromatosis? AJR Am J Roentgenol 200:W646-653, 2013

11. Korf BR: Neurofibromatosis. Handb Clin Neurol 111:333340,2013

12. MacCollin M, Chiocca EA, Evans DG, Friedman JM, Horvitz R, Jaramillo D, et al: Diagnostic criteria for schwannomatosis. Neurology 64:1838-1845, 2005

13. Mannan AASR, Singh MK, Bahadur S, Hatimota P, Sharma MC: Solitary malignant schwannoma of the nasal cavity and paranasal sinuses: report of two rare cases. Ear Nose Throat J 82:634-636, 638, 640, 2003

14. Merker VL, Esparza S, Smith MJ, Stemmer-Rachamimov A, Plotkin SR: Clinical features of schwannomatosis: a retrospective analysis of 87 patients. Oncologist 17:1317-1322, 2012
15. Paganini I, Chang VY, Capone GL, Vitte J, Benelli M, Barbetti L, et al: Expanding the mutational spectrum of LZTR1 in schwannomatosis. Eur J Hum Genet 23:963-968, 2015

16. Piotrowski A, Xie J, Liu YF, Poplawski AB, Gomes AR, Madanecki P, et al: Germline loss-of-function mutations in LZTR1 predispose to an inherited disorder of multiple schwannomas. Nat Genet 46:182-187, 2014

17. Rizzo D, Fréneaux P, Brisse H, Louvrier C, Lequin D, Nicolas A, et al: SMARCB1 deficiency in tumors from the peripheral nervous system: a link between schwannomas and rhabdoid tumors? Am J Surg Pathol 36:964-972, 2012

18. Rodriguez FJ, Stratakis CA, Evans DG: Genetic predisposition to peripheral nerve neoplasia: diagnostic criteria and pathogenesis of neurofibromatoses, Carney complex, and related syndromes. Acta Neuropathol 123:349-367, 2012

19. Sestini R, Bacci C, Provenzano A, Genuardi M, Papi L: Evidence of a four-hit mechanism involving SMARCB1 and NF2 in schwannomatosis-associated schwannomas. Hum Mutat 29:227-231, 2008

20. Sheikh HY, Chakravarthy RP, Slevin NJ, Sykes AJ, Banerjee SS: Benign schwannoma in paranasal sinuses: a clinicopathological study of five cases, emphasising diagnostic difficulties. J Laryngol Otol 122:598-602, 2008

21. Smith MJ, Wallace AJ, Bowers NL, Rustad CF, Woods CG, Leschziner GD, et al: Frequency of SMARCB1 mutations in familial and sporadic schwannomatosis. Neurogenetics 13:141-145, 2012

22. Smith MJ, Wallace AJ, Bowers NL, Eaton H, Evans DGR: SMARCB1 mutations in schwannomatosis and genotype correlations with rhabdoid tumors. Cancer Genet 207:373-378, 2014

23. Yoshizawa M, Sugita K, Haruyama S, Yoshiki R, Hino R, Bito T, et al: Schwannomatosis presenting with large subcutaneous and retroperitoneal tumours. Clin Exp Dermatol 36:555-557, 2011

24. Zhang K, Lin JW, Wang J, Wu X, Gao H, Hsieh YC, et al: A germline missense mutation in COQ6 is associated with susceptibility to familial schwannomatosis. Genet Med 16:787-792, 2014

\section{Disclosure}

The authors report no conflict of interest concerning the materials or methods used in this study or the findings specified in this paper.

\section{Author Contributions}

Conception and design: Toms, Harrison, Richard, Graham. Acquisition of data: all authors. Analysis and interpretation of data: Toms, Harrison, Graham. Drafting the article: Toms, Harrison, Richard. Critically revising the article: Toms, Harrison. Reviewed submitted version of manuscript: Toms, Harrison, Graham. Approved the final version of the manuscript on behalf of all authors: Toms. Administrative/technical/material support: Toms. Study supervision: Graham.

\section{Correspondence}

Jamie Toms, VCU Medical Center-Ambulatory Care Center, 6th Fl., P.O. Box 980631, Richmond, VA 23298. email: jtoms@ mcvh-vcu.edu. 